\title{
Case Report \\ Implantation of collagen punctal plugs for the reduction of post-keratoplasty ametropia
}

\author{
Baskin D, Waller S \\ Wilford Hall Medical Center, San Antonio, Texas 78209, USA
}

\begin{abstract}
In 1995, a 46-year-old woman was referred for refractive surgery. Eleven years earlier, she had undergone an uncomplicated penetrating keratoplasty in her right eye to treat a dense corneal opacity caused by contact lens-related microbial keratitis. Due to high myopia in the graft, she was unable to achieve useful vision and declined contact lens use. After extensive counseling, she elected to undergo radial keratotomy (RK) and arcuate keratotomy (AK) in her right eye. We recommended the insertion of collagen punctal plugs into the keratotomy incisions to increase the RK effect. She also decided to have RK in her left eye. Postoperatively, her uncorrected visual acuity was 20/25 OD and 20/30 OS. We were able to contact her and her ophthalmologist eleven years later, and eight of eleven collagen plugs were still present in the incisions, with no signs of rejection or inflammation. Later, we learned that she underwent a repeat penetrating keratoplasty due to corneal graft failure after a phacoemulsification procedure. The pathologic examination of the intact plugs and graft also showed no rejection or inflammation.
\end{abstract}

\section{History}

In 1995, a 46-year-old woman was referred to Wilford Hall Medical Center (WHMC) for refractive surgery twelve years after an uncomplicated penetrating keratoplasty (PKP) in her right eye. The PKP had been performed after a corneal opacity developed due to contact lens-related pseudomonas keratitis. At her initial presentation to WHMC, the manifest refraction of her right eye exceeded the predicted maximum amount of myopia that radial keratotomy (RK) would treat, according to the eight-incision RK Casebeer nomogram (Papaioannou et al 1997). In an effort to correct the astigmatism and additional myopia in her right eye, and after an extensive discussion of the risks, benefits and alternatives, she elected to undergo RK and arcuate keratotomy (AK) with the insertion of collagen punctal plugs into the keratotomy incisions.

Received: 26.09.2008. Accepted: 07.10.2008

Correspondence and reprint requests to: Darrell Baskin, MD, and Stephen Waller, MD

Department of Ophthalmology

Wilford Hall Medical Center

San Antonio, Texas 78209

USA
In the right eye, she underwent two arcuate incisions at $4 \mathrm{~mm}$ on the $35^{\circ}$ axis followed by nine bi-directional, radial incisions at a $3.75 \mathrm{~mm}$ optical zone (OZ). One collagen punctal plug was then placed in each of the eleven incisions. There were no complications. In the left eye, she later underwent eight bi-directional, radial incisions at a $3.75 \mathrm{~mm} \mathrm{OZ}$ without complications.

\section{Examination}

On postoperative day one examination, the uncorrected visual acuity was 20/25 in the right eye and 20/30 in the left eye. Ocular motility, pupil reactivity, confrontational visual fields and intraocular pressures were within normal limits. The slit-lamp examination of the right eye revealed a quiet conjunctiva and the cornea had two arcuate incisions and nine radial incisions, each of which had a collagen punctal plug buried within it (Figure 1). Over the course of the next three months, three of the collagen plugs spontaneously extruded from the 1:00 and 3:00 o'clock RK incisions, and the 2:00 o'clock AK incision. The rest of the collagen plugs remained within the incisions, and none of the incisions showed infiltrates, edema, or other corneal abnormalities. 



Figures $1 \& 2$. Slit-lamp photographs of right eye (Fig. 1 is 1 week after surgery; Fig. 2 is 9 months after surgery) demonstrating collagen plugs within some of the keratotomy incisions.

Nine months after the procedure, corneal topography was performed on the right eye, revealing simulated keratometry values of 47.66 D@042,45.73 D@132 (Figure 3).

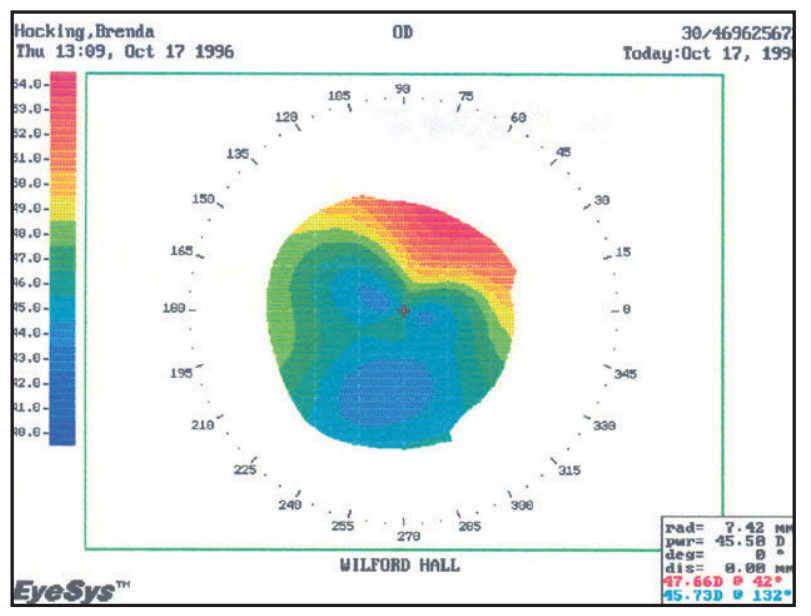

Figure 3. Corneal topography of right eye after RK and AK with collagen plugs in 8 of 11 incisions demonstrating irregular astigmatism with a steep axis around $67^{\circ}$.

The patient subsequently moved to another state and received regular ophthalmic care from a local ophthalmologist.

In 2006, the patient presented to her local ophthalmologist with a chief complaint of blurry vision OD. She was found to have a visually-significant cataract. She elected to undergo phacoemulsification and intraocular lens placement. She had preoperative and postoperative corneal topography performed, as well as Pentacam computed optical tomography done postoperatively (Figures 4-6). The simulated keratometry readings from the Pentacam scan were 56.87D@116,44.37 D@026.

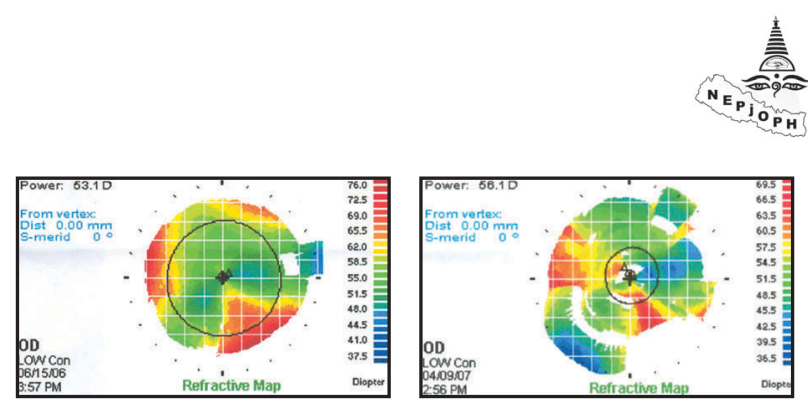

Figures $4 \& 5$. Refractive maps of the right eye. Figure 4 was 2 weeks before phacoemulsification, and Figure 5 represents 10 months after phacoemulsification.



Figure 6. Bright flash (Scheimpflug) image of right eye.

After phacoemulsification, the corneal graft became ectatic, and in 2007, she underwent another penetrating keratoplasty. The corneal tissue was sent to pathology (Figure 7).

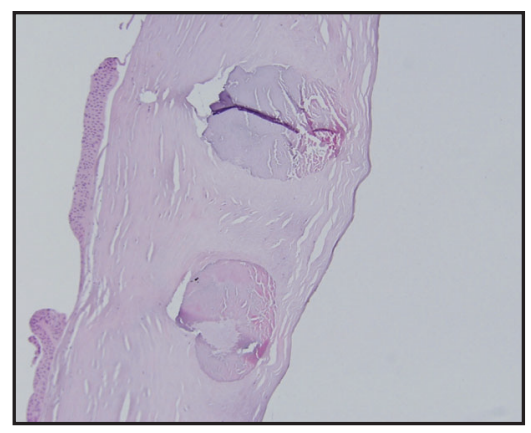

Figure 7. Corneal graft, right eye. Hematoxylin and eosin stain at $10 \mathrm{x}$ magnification, demonstrating collagen plugs embedded within keratotomy incisions without inflammation.

\section{Discussion and diagnosis}

Our patient had post-keratoplasty anisometropia before photoablative procedures were widely used in the USA. She refused to try a contact lens because of her previous microbial keratitis, and she was seeking improved visual function in an eye that was otherwise severely disabled. Because the published nomograms for radial keratotomy predicted her high myopia was only partially treatable, she was offered the off-label use of an ophthalmic device (FDA-approved for punctal occlusion) to theoretically increase the corneal flattening effect. 
It was hypothesized that the implantation of the collagen plugs into the keratotomy incisions would increase the incisional gape and thus extend the range of the RK nomogram. One of the authors (SGW) had recently studied the effect of collagen implants placed within incisional keratotomies in feline and rabbit eyes, which did not demonstrate clear enhancement of the RK effect, but did appear to be safe in the two live animal models (Waller et al 1996). The principal considerations included the fact that the anticipated eight-incision effect would have been sub-therapeutic and the incision lengths would have to be shorter in order to avoid cutting through the host-graft interface. Finally, the nomogram was not designed for postkeratoplasty eyes and surgery would likely yield less myopic correction due to the bolstering effect of the graft.

After phacoemulsification, her corneal graft became ectatic and failed. It is possible that the collagen plugs may have contributed to this event. The collagen plugs were otherwise well-tolerated and did not promote any clinically-apparent graft rejection or other corneal inflammatory event. All of the collagen plugs that were in place 9 months after placement ( 8 of 11) remained within the incisions permanently. This clinical finding was confirmed on pathologic examination of the tissue.

\section{Conclusion}

The management of post-keratoplasty ametropia and astigmatism has evolved drastically since the advent of the photoablative corneal refractive procedures. Post-keratoplasty laser-assisted in situ keratomileusis has been very successful in the treatment of both astigmatism and spherical errors (Hardten et al 2004; Malecha et al 2002). Photorefractive keratectomy has also been used in the treatment of post-keratoplasty astigmatism and myopia, but Bilgihan et al (2000) noted that this technique is sometimes beset by excessive corneal haze. Prior to the photoablative period, however, there were more limited options in the treatment of post-keratoplasty ametropia, and the use of collagen plugs in this patient was a unique approach in the management of a difficult problem. In the literature, the only other use of corneal collagen plugs was within AK incisions in the treatment of postkeratoplasty astigmatism, which yielded some documented benefit (Espaillat et al 1999). The apparent long-term safety and efficacy of this single case of collagen plugs implanted within RK and AK incisions may have value for surgeons who do not have easy access to a refractive laser. This study may also have implications for further studies of corneal tectonics and ectasia.

\section{References}

Bilighan K, Ozdek SC, Akata F et al (2000). Photorefractive keratectomy for post-penetrating keratoplasty myopia and astigmatism. J Cataract Refract Surg; 26(11):1590-1595

Espaillat A, To K, Perlman EM (1999). Effect of collagen implants in the reduction of high astigmatism induced by penetrating keratoplasty. J Cataract Refract Surg; 25:1336-1340.

Hardten DR, Chittcharus A, Lindstrom RL (2004). Long term analysis of LASIK for the correction of refractive errors after penetrating keratoplasty. Cornea; 23(5):479-489.

Malecha MA, Holland EJ (2002). Correction of myopia and astigmatism after penetrating keratoplasty with laser in-situ keratomileusis. Cornea; 21(6):564-569.

Papaioannou T, Salz, JJ, and Maguen E (1997). Predictive formulas for refractive surgery. In Cornea, Krachmer JH, Mannis MJ, and Holland EJ. (eds), vol III, ch. 164, pp 2025-2037, Mosby-Yearbook, St. Louis.

Waller SG, LaMarche K, and Talamo JH (1996). Implantation of collagen plugs into incisional keratotomies. J Cataract Refract Surg; 22(4):483491. 